\title{
Uniform Statistical Convergence on Time Scales
}

\author{
Yavuz Altin, Hikmet Koyunbakan, and Emrah Yilmaz \\ Department of Mathematics, Firat University, 23119 Elazı, Turkey \\ Correspondence should be addressed to Emrah Yilmaz; emrah231983@gmail.com
}

Received 22 March 2014; Accepted 30 May 2014; Published 18 June 2014

Academic Editor: Alberto Cabada

Copyright (C) 2014 Yavuz Altin et al. This is an open access article distributed under the Creative Commons Attribution License, which permits unrestricted use, distribution, and reproduction in any medium, provided the original work is properly cited.

We will introduce the concept of $m$ - and $(\lambda, m)$-uniform density of a set and $m$ - and $(\lambda, m)$-uniform statistical convergence on an arbitrary time scale. However, we will define $m$-uniform Cauchy function on a time scale. Furthermore, some relations about these new notions are also obtained.

\section{Introduction}

The idea of statistical convergence was known to Zygmund [1] as early as 1935 and in particular after 1951 when Fast [2] and Steinhaus [3] reintroduced statistical convergence for sequences of real numbers. Later, Schoenberg [4] independently gave some basic properties of statistical convergence. Several generalizations and applications of this notion have been discussed in the theory of Fourier analysis, ergodic theory, number theory, measure theory, trigonometric series, turnpike theory, and Banach spaces under different names (see [5-11]).

Statistical convergence depends on the density of subsets of the set $\mathbb{N}$. Recall that a subset $A$ of $\mathbb{N}$ is said to have "asymptotic density" $\delta(A)$ if

$$
\delta(A)=\lim _{n \rightarrow \infty} \frac{1}{n}|\{k \leq n: k \in A\}|,
$$

where the vertical bars denote the cardinality of the enclosed set. It is clear that any finite subset of $\mathbb{N}$ has zero asymptotic density and $\delta\left(A^{c}\right)=1-\delta(A)$ (see [12]).

A sequence $\left\{x_{k}\right\}_{k \in \mathbb{N}}$ is said to be statistically convergent to a real number $L$ if

$$
\lim _{n \rightarrow \infty} \frac{1}{n}\left|\left\{k \leq n:\left|x_{k}-L\right| \geq \varepsilon\right\}\right|=0,
$$

for each $\varepsilon>0$. And we write $x_{k}$ st $L$ or $S-\lim x_{k}=L$. The set of all statistically convergent sequences is denoted by $S$ (see $[2,3,5,6,9,13])$.
The generalized de la Vallée-Poussin mean is defined by

$$
t_{n}(x)=\frac{1}{\lambda_{n}} \sum_{k \in I_{n}} x_{k},
$$

where $\lambda=\left(\lambda_{n}\right)$ is a nondecreasing sequence of positive numbers such that $\lambda_{n+1} \leq \lambda_{n}+1, \lambda_{1}=1, \lambda_{n} \rightarrow \infty$ as $n \rightarrow \infty$ and $I_{n}=\left[n-\lambda_{n}+1, n\right]$. The set of all such sequences will be denoted by $\Lambda$ (see [14]).

A sequence $x=\left(x_{k}\right)$ is said to be $(V, \lambda)$-summable to a number $L$ if $t_{n}(x) \rightarrow L$ as $n \rightarrow \infty$. $(V, \lambda)$-summability reduces to $(C, 1)$ summability when $\lambda=\left(\lambda_{n}\right)=(n)$ (see [14]).

We write

$[C, 1]=\left\{x=\left(x_{k}\right): \lim _{n \rightarrow \infty} \frac{1}{n} \sum_{k=1}^{n}\left|x_{k}-L\right|=0\right.$ for some $\left.L\right\}$,

$[V, \lambda]=\left\{x=\left(x_{k}\right): \lim _{n \rightarrow \infty} \frac{1}{\lambda_{n}} \sum_{k \in I_{n}}\left|x_{k}-L\right|=0\right.$ for some $\left.L\right\}$,

for the sets of sequences $x=\left(x_{k}\right)$ which are strongly Cesàro summable and strongly $(V, \lambda)$-summable, respectively. Strong $(V, \lambda)$-summability reduces to strong $(C, 1)$ summability when $\lambda_{n}=n$.

The notion of $\lambda$-statistical convergence was introduced by Mursaleen [15] as follows.

Let $K \subset \mathbb{N}$ and define the $\lambda$-density of $K$ by

$$
\delta_{\lambda}(K)=\lim _{n \rightarrow \infty} \frac{1}{\lambda_{n}}\left|\left\{n-\lambda_{n}+1 \leq k \leq n: k \in K\right\}\right| .
$$


$\delta_{\lambda}(K)$ reduces to the asymptotic density $\delta(K)$ in case of $\lambda_{n}=$ $n$ for all $n \in \mathbb{N}$ (see [15]).

A sequence $x=\left(x_{k}\right)$ is said to be $\lambda$-statistically convergent to $L$ if for every $\varepsilon>0$ (see [15])

$$
\lim _{n \rightarrow \infty} \frac{1}{\lambda_{n}}\left|\left\{k \in I_{n}:\left|x_{k}-L\right| \geq \varepsilon\right\}\right|=0 .
$$

After the concept of almost $\lambda$-statistical convergence was studied by Savaş [16], many authors have studied statistical convergence (see $[6,9,10]$ ).

Statistical convergence is applied to time scales for different purposes by various authors (see $[17,18]$ ).

We here recall some basic concepts and notations from the theory of time scales. A time scale is an arbitrary nonempty closed set of real numbers. We use the symbol $\mathbb{T}$ to denote a time scale. A time scale has the topology that it inherits from the real numbers with the standard topology. The theory of time scale was introduced by Hilger in his Ph.D. thesis supervised by Auldbach in 1988 (see [19]). It allows unifying the usual differential and integral calculus for one variable. One can replace the range of definition $(\mathbb{R})$ of the functions under consideration by an arbitrary time scale $\mathbb{T}$. Now, time scale theory has been applied to different areas by many authors (see [20-24]).

The forward jump operator $\sigma: \mathbb{T} \rightarrow \mathbb{T}$ can be defined by

$$
\sigma(t)=\inf \{s \in \mathbb{T}: s>t\},
$$

for $t \in \mathbb{T}$. And the graininess function $\mu: \mathbb{T} \rightarrow[0, \infty)$ is defined by $\mu(t)=\sigma(t)-t$. In this definition, we put inf $\phi=$ sup $\mathbb{T}$, where $\phi$ is an empty set. A half open interval on an arbitrary time scale $\mathbb{T}$ is given by

$$
[a, b)_{\mathbb{T}}=\{t \in \mathbb{T}: a \leq t<b\} .
$$

Open intervals or closed intervals can be defined similarly (see $[20,21])$.

Now, let $A$ denote the family of all left closed and right open intervals of $\mathbb{T}$ of the form $[a, b)_{\mathbb{T}}$. Let $s: A \rightarrow[0, \infty)$ be the set function on $A$ such that

$$
s\left([a, b)_{\mathbb{T}}\right)=b-a .
$$

Then, it is known that $s$ is a countably additive measure on $A$. Now, the Caratheodory extension of the set function $s$ associated with family $A$ is said to be the Lebesgue $\Delta$-measure on $\mathbb{T}$ and is denoted by $\mu_{\Delta}$. In this case, it is known that if $a \in \mathbb{T}-\{\max \mathbb{T}\}$, then the single point set $\{a\}$ is $\Delta$-measurable and $\mu_{\Delta}(a)=\sigma(a)-a$. If $a, b \in \mathbb{T}$ and $a \leq b$, then $\mu_{\Delta}\left((a, b)_{\mathbb{T}}\right)=$ $b-\sigma(a)$. If $a, b \in \mathbb{T}-\{\max \mathbb{\mathbb { V }}\}, a \leq b ; \mu_{\Delta}\left((a, b]_{\mathbb{V}}\right)=\sigma(b)-\sigma(a)$ and $\left.\mu_{\Delta}([a, b])_{\mathbb{V}}\right)=\sigma(b)-a($ see $[18])$.

In this study, we will give some notations for $m$-uniform and $(\lambda, m)$-uniform density of a set and $m$-uniform and $(\lambda, m)$-uniform statistical convergence and some properties of $m$-uniform and $(\lambda, m)$-uniform statistical convergence on time scales.

Definition 1 (see [25]). A subset $E$ of $\mathbb{N}$ is said to be uniformly dense if

$$
u(E)=\lim _{n \rightarrow \infty} \frac{1}{n} \sum_{j=1}^{n} \chi_{E}(j+m)=a
$$

uniformly in $m$ or, equivalently,

$$
\lim _{n \rightarrow \infty} \frac{1}{n}|E \cap\{m+1, \ldots, m+n\}|=a,
$$

uniformly in $m$, where $m=0,1,2,3, \ldots$ and $\chi_{E}$ is characteristic function. Subsequently, uniform density was studied by Baláž and Šalát [26], Brown and Freedman [27], and Maddox [28].

The notion of $m$-uniform statistical convergence is first introduced by Nuray [29] as follows.

Definition 2 (see [29]). Let $x=\left(x_{k}\right)$ be a real or complex valued sequence. If

$$
\lim _{n \rightarrow \infty} \frac{1}{n}\left|\left\{m \leq k<n+m:\left|x_{k}-L\right| \geq \varepsilon\right\}\right|=0
$$

uniformly in $m, x=\left(x_{k}\right)$ is said to be $m$-uniform statistically convergence to $L$ for $\varepsilon>0$.

Based on this notion, we give the following definitions to generalize $m$-uniform statistical convergence.

Definition 3. Let $K \subset \mathbb{N}$ and define the $(\lambda, m)$-uniform density of $K$ by

$$
\delta_{\lambda}^{m}(K)=\lim _{n \rightarrow \infty} \frac{1}{\lambda_{n}}\left|\left\{n+m-\lambda_{n} \leq k<n+m: k \in K\right\}\right| .
$$

$\delta_{\lambda}^{m}(K)$ reduces to the $\delta^{m}(K)$ in case of $\lambda_{n}=n$ for all $n \in \mathbb{N}$.

Definition 4. A sequence $x=\left(x_{k}\right)$ is said to be $(\lambda, m)$ uniform statistically convergent to $L$ if

$$
\lim _{n \rightarrow \infty} \frac{1}{\lambda_{n}}\left|\left\{n+m-\lambda_{n} \leq k<n+m:\left|x_{k}-L\right| \geq \varepsilon\right\}\right|=0
$$

for every $\varepsilon>0$ uniformly in $m$.

In [30], Borwein introduced and studied strongly summable functions. His definition is as follows.

Definition 5 (see [30]). A real-valued function $x(t)$, measurable (in Lebesgue sense) on the interval $(1, \infty)$, is said to be strongly summable to $L=L_{x}$ if

$$
\lim _{n \rightarrow \infty} \frac{1}{n} \int_{1}^{n}|x(t)-L|^{p} d t=0, \quad 1 \leq p<\infty .
$$

$\left[W_{p}\right]$ will denote the space of real-valued function $x$, measurable (in the Lebesgue sense) on the interval $(1, \infty)$.

Furthermore, Nuray [31] studied $\lambda$-strong summable and $\lambda$-statistically convergent functions as in the following.

Definition 6 (see [31]). Let $\lambda \in \Lambda$, let $p$ be a real number, and let $x(t)$ be a real-valued function which is measurable (in Lebesgue sense) on the interval $(1, \infty)$, if

$$
\lim _{n \rightarrow \infty} \frac{1}{\lambda_{n}} \int_{n-\lambda_{n}+1}^{n}|x(t)-L|^{p} d t=0 ;
$$

then, one says that $x(t)$ is $\lambda_{p}$ strongly summable to $L$. Strongly summable number sequences and statistically convergent 
number sequences were studied by Maddox [32], Nuray and Aydin [33], and Et et al. [34].

There are some studies about statistical convergence on time scales in the literature. For instance, Seyyidoglu and Tan [17] gave some new notations such as $\Delta$-convergence and $\Delta$ Cauchy by using $\Delta$-density and investigated their relations. Turan and Duman [18] introduced the concept of density and statistical convergence of delta measurable real-valued functions defined on time scales as follows.

Definition 7 (see [18]). Suppose that $\Omega$ is a $\Delta$-measurable subset of $\mathbb{T}$. Then, for $t \in \mathbb{T}$, one defines the set $\Omega(t)$ by

$$
\Omega(t)=\left\{s \in\left[t_{0}, t\right]_{\mathbb{T}}: s \in \Omega\right\}
$$

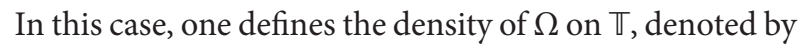
$\delta_{\mathbb{T}}(\Omega)$,

$$
\delta_{\mathbb{T}}(\Omega)=\lim _{t \rightarrow \infty} \frac{\mu_{\Delta}(\Omega)}{\mu_{\Delta}\left(\left[t_{0}, t\right]_{\mathbb{T}}\right)},
$$

provided that the above limit exists. Furthermore, $f$ is statistically convergent to a real number $L$ on $\mathbb{T}$ if, for every $\varepsilon>0$

$$
\delta_{\mathbb{T}}(\{t \in \mathbb{T}:|f(t)-L| \geq \varepsilon\})=0,
$$

where $f: \mathbb{T} \rightarrow \mathbb{R}$ is a $\Delta$-measurable function (see $[17,18]$ ). Lebesgue $\Delta$-measure $\mu_{\Delta}$ is introduced by Guseinov [20].

Definition 8 (see [18]). Let $f: \mathbb{T} \rightarrow \mathbb{R}$ be a $\Delta$-measurable function. $f$ is statistical Cauchy on $\mathbb{\mathbb { T }}$ if, for each $\varepsilon>0$, there exists a number $t_{1}>t_{0} \in \mathbb{T}$ such that

$$
\lim _{t \rightarrow \infty} \frac{\mu_{\Delta}\left(\left\{s \in\left[t_{0}, t\right]_{\mathbb{T}}:\left|f(s)-f\left(t_{1}\right)\right| \geq \varepsilon\right\}\right)}{\mu_{\Delta}\left(\left[t_{0}, t\right]_{\mathbb{T}}\right)}=0 .
$$

\section{Main Results and Preliminaries}

It is well known that the notion of statistical convergence is closely related to the density of the subset of $\mathbb{N}$. So, in this section, we will first define $m$-uniform and $(\lambda, m)$ uniform density of the subset of the time scale. By using these definitions, we will focus on constructing a concept of $m$-uniform (or $(\lambda, m)$-uniform) statistical convergence and $m$-uniform statistical Cauchy function on time scales. In following definitions, notations $\Delta_{m}$ and $\Delta_{(\lambda, m)}$ shows that $\Delta$ depends on $m$ and $(\lambda, m)$, respectively.

Definition 9. Let $\Omega$ be a $\Delta_{m}$-measurable subset of $\mathbb{T}$. Then, one defines the set $\Omega(t, m)$ by

$$
\Omega(t, m)=\left\{s \in\left[m+t_{0}-1, t+m\right): s \in \Omega\right\},
$$

for $t \in \mathbb{T}$. In this case, one defines the $m$-uniform density of $\Omega$ on $\mathbb{T}$, denoted by $\delta_{\mathbb{T}}^{m}(\Omega)$, as follows:

$$
\delta_{\mathbb{T}}^{m}(\Omega)=\lim _{t \rightarrow \infty} \frac{\mu_{\Delta_{m}}(\Omega(t, m))}{\mu_{\Delta_{m}}\left(\left[m+t_{0}-1, t+m\right)_{\mathbb{T}}\right)},
$$

provided that the above limit exists.
Definition 10. Let $f: \mathbb{T} \rightarrow \mathbb{R}$ be a $\Delta_{m}$-measurable function. Then, one says that $f$ is $m$-uniform statistically convergent to a real number $L$ on $\mathbb{T}$ if

$$
\lim _{t \rightarrow \infty} \frac{\mu_{\Delta_{m}}\left(s \in\left[m+t_{0}-1, t+m\right):|f(s)-L| \geq \varepsilon\right)}{\mu_{\Delta_{m}}\left(\left[m+t_{0}-1, t+m\right)_{\mathbb{T}}\right)}=0,
$$

uniformly in $m$ for every $\varepsilon>0$. In this case, one writes $\widehat{\mathbf{s}}_{\mathbb{T}}^{m}-\lim _{t \rightarrow \infty}(f(t))=L$. The set of all $m$-uniform statistically convergent functions on $\mathbb{T}$ will be denoted by $\widehat{\mathbf{s}}_{\mathbb{T}}^{m}$.

In case of $\mathbb{T}=\mathbb{N},\left[m+t_{0}-1, t+m\right)$ is $[m, n+m)$ for $t=n$ and $t_{0}=1$. In this instance, $m$-uniform statistical convergence on time scales is reduced to classical $m$-uniform statistical convergence which is given by Definition 2. This shows that our results are generalizations of classical results.

Similarly, we can define $m$-uniform statistical Cauchy functions on a time scale based on Definition 8 .

Definition 11. Let $f: \mathbb{T} \rightarrow \mathbb{R}$ be a $\Delta_{m}$-measurable function. $f$ is an $m$-uniform statistical Cauchy function on $\mathbb{T}$ if there exists a number $t_{1}>t_{0} \in \mathbb{T}$ such that

$$
\lim _{t \rightarrow \infty} \frac{\mu_{\Delta_{m}}\left(\left\{s \in\left[m+t_{0}-1, t+m\right)_{\mathbb{T}}:\left|f(s)-f\left(t_{1}\right)\right| \geq \varepsilon\right\}\right)}{\mu_{\Delta_{m}}\left(\left[m+t_{0}-1, t+m\right)_{\mathbb{T}}\right)}
$$

$=0$

for each $\varepsilon>0$ uniformly in $m$. One can easily see that this definition is a generalization of Definition 8.

Definition 12. Let $\Omega(t, m, \lambda)$ be a $\Delta_{(\lambda, m)}$-measurable subset of $\mathbb{T}$. Then, one defines the set $\Omega(t, m, \lambda)$ by

$$
\Omega(t, m, \lambda)=\left\{s \in\left[t+m-\lambda_{t}+t_{0}-1, t+m\right): s \in \Omega\right\}
$$

for $t \in \mathbb{T}$. In this case, one defines the $(\lambda, m)$-uniform density of $\Omega$ on $\mathbb{T}$ denoted by $\delta_{\mathbb{T}}^{(\lambda, m)}(\Omega)$, as follows:

$$
\delta_{\mathbb{T}}^{(\lambda, m)}(\Omega)=\lim _{t \rightarrow \infty} \frac{\mu_{\Delta_{(\lambda, m)}}(\Omega(t, m, \lambda))}{\mu_{\Delta_{(\lambda, m)}}\left(\left[t+m-\lambda_{t}+t_{0}-1, t+m\right)_{\mathbb{T}}\right)},
$$

provided that the above limit exists.

Definition 13. Let $f: \mathbb{T} \rightarrow \mathbb{R}$ be a $\Delta_{(\lambda, m)}$-measurable function. One says that $f$ is $(\lambda, m)$-uniform statistically convergent to a real number $L$ on $\mathbb{T}$ if

$$
\lim _{t \rightarrow \infty} \frac{\mu_{\Delta_{(\lambda, m)}}\left(s \in\left[t+m-\lambda_{t}+t_{0}-1, t+m\right):|f(s)-L| \geq \varepsilon\right)}{\mu_{\Delta_{(\lambda, m)}}\left(\left[t+m-\lambda_{t}+t_{0}-1, t+m\right)_{\mathbb{T}}\right)}
$$$$
=0
$$

uniformly in $m$ for every $\varepsilon>0$. In this case, one writes $\widehat{\mathbf{s}}_{\mathbb{T}}^{(\lambda, m)}-$ $\lim _{t \rightarrow \infty}(f(t))=L$. The set of all $(\lambda, m)$-uniform statistically convergent functions on $\mathbb{T}$ will be denoted by $\widehat{\mathbf{s}}_{\mathbb{T}}^{(\lambda, m)}$. 
Hence, we have generalized Definition 3 to an arbitrary time scale. We can easily get classical $(\lambda, m)$-uniform statistical convergence by taking $t_{0}=1$ in Definition 13 .

Proposition 14. If $f, g: \mathbb{T} \rightarrow \mathbb{R}$ with $\widehat{\mathbf{s}}_{\mathbb{T}}^{(\lambda, m)}-\lim _{t \rightarrow \infty} f(t)=$ $L_{1}$ and $\widehat{\mathbf{s}}_{\mathbb{T}}^{(\lambda, m)}-\lim _{t \rightarrow \infty} g(t)=L_{2}$, then the following statements hold:

(i) $\widehat{\mathbf{s}}_{\mathbb{T}}^{(\lambda, m)}-\lim _{t \rightarrow \infty}(f(t)+g(t))=L_{1}+L_{2}$,

(ii) $\widehat{\mathbf{s}}_{\mathbb{T}}^{(\lambda, m)}-\lim _{t \rightarrow \infty}(c f(t))=c L_{1}(c \in \mathbb{R})$.

Theorem 15. For $f: \mathbb{T} \rightarrow \mathbb{R}$ to be any $\Delta_{(\lambda, m)}$-measurable function, $f$ is $(\lambda, m)$-uniform statistically convergent on $\mathbb{T}$ if and only if $f$ is a $(\lambda, m)$-uniform statistical Cauchy function on $\mathbb{T}$.

Proof. We can prove this by using techniques similar to Theorem 3 of [29].

Theorem 16. Consider $\widehat{\mathbf{s}}_{\mathbb{T}}^{m} \subset \widehat{\mathbf{s}}_{\mathbb{U}}^{(\lambda, m)}$ if and only if

$$
\lim _{t \rightarrow \infty} \inf \frac{\mu_{\Delta_{(\lambda, m)}}\left(\left[t+m-\lambda_{t}+t_{0}-1, t+m\right)_{\mathbb{T}}\right)}{\mu_{\Delta_{m}}\left(\left[m+t_{0}-1, t+m\right)_{\mathbb{T}}\right)}>0 .
$$

Proof. For given $\varepsilon>0$, we have

$$
\begin{gathered}
\mu_{\Delta_{m}}\left(s \in\left[m+t_{0}-1, t+m\right)_{\mathbb{T}}:|f(s)-L| \geq \varepsilon\right) \\
\supset \mu_{\Delta_{(\lambda, m)}}\left(s \in\left[t+m-\lambda_{t}+t_{0}-1, t+m\right)_{\mathbb{T}}:\right. \\
|f(s)-L| \geq \varepsilon) .
\end{gathered}
$$

Therefore,

$$
\begin{aligned}
& \left(\mu_{\Delta_{m}}\left(s \in\left[m+t_{0}-1, t+m\right)_{\mathbb{T}}:|f(s)-L| \geq \varepsilon\right)\right) \\
& \quad \times\left(\mu_{\Delta_{m}}\left(\left[m+t_{0}-1, t+m\right)_{\mathbb{T}}\right)\right)^{-1} \\
& \geq\left(\mu _ { \Delta _ { ( \lambda , m ) } } \left(s \in\left[t+m-\lambda_{t}+t_{0}-1, t+m\right)_{\mathbb{T}}:\right.\right. \\
& \quad \times\left(\mu_{\Delta_{m}}\left(\left[m+t_{0}-1, t+m\right)_{\mathbb{T}}\right)\right)^{-1} \\
& =\frac{\mu_{\Delta_{(\lambda, m)}}\left(\left[t+m-\lambda_{t}+t_{0}-1, t+m\right)_{\mathbb{J}}\right)}{\mu_{\Delta_{m}}\left(\left[m+t_{0}-1, t+m\right)_{\mathbb{J}}\right)} \\
& \quad \times \frac{1}{\mu_{\Delta_{(\lambda, m)}}\left(\left[t+m-\lambda_{t}+t_{0}-1, t+m\right)_{\mathbb{T}}\right)} \\
& \quad \times \mu_{\Delta_{(\lambda, m)}}\left(s \in\left[t+m-\lambda_{t}+t_{0}-1, t+m\right)_{\mathbb{T}}:\right. \\
& |f(s)-L| \geq \varepsilon) .
\end{aligned}
$$

Hence by using (28) and taking the limit as $t \rightarrow \infty$, we get $f(s) \rightarrow L\left(\widehat{\mathbf{s}}_{\mathbb{T}}^{m}\right)$ which implies $f(s) \rightarrow L\left(\widehat{\mathbf{s}}_{\mathbb{T}}^{(\lambda, m)}\right)$.

The definition of $p$-Cesàro summability on time scales was given by Turan and Duman [18] as follows.
Definition 17 (see [18]). Let $f: \mathbb{T} \rightarrow \mathbb{R}$ be a $\Delta$-measurable function and $0<p<\infty$. Then, $f$ is strongly $p$-Cesàro summable on $\mathbb{T}$ if there exists some $L \in \mathbb{R}$ such that

$$
\lim _{t \rightarrow \infty} \frac{1}{\mu_{\Delta}\left(\left[t_{0}, t\right]_{\mathbb{T}}\right)} \int_{\left[t_{0}, t\right]_{\mathbb{T}}}|f(s)-L|^{p} \Delta s=0 .
$$

The set of all $p$-Cesàro summable functions on $\mathbb{T}$ will be denoted by $\left[W_{p}\right]_{T}$.

Measure theory on time scales was first constructed by Guseinov [20] and Lebesgue $\Delta$-integral on time scales introduced by Cabada and Vivero [35].

Definition 18. Let $f: \mathbb{T} \rightarrow \mathbb{R}$ be a $\Delta_{(\lambda, m)}$-measurable function and $0<p<\infty$. One says that $f$ is $(\lambda, m)$ uniformly strongly $p$-summable on $\mathbb{T}$ if there exists some $L \in \mathbb{R}$ such that

$$
\begin{aligned}
& \lim _{t \rightarrow \infty} \frac{1}{\mu_{\Delta_{(\lambda, m)}}\left(\left[t+m-\lambda_{t}+t_{0}-1, t+m\right)_{\mathbb{T}}\right)} \\
& \quad \times \int_{\left[t+m-\lambda_{t}+t_{0}-1, t+m\right)_{\mathbb{T}}}|f(s)-L|^{p} \Delta s=0 .
\end{aligned}
$$

In this case, one writes $\left[\widehat{W}_{m p}\right]_{\mathbb{T}}-\lim f(s)=L$. The set of all $(\lambda, m)$ uniformly strongly $p$-summable functions on $\mathbb{T}$ will be denoted by $\left[\widehat{W}_{m p}\right]_{\mathbb{T}}$.

Lemma 19. Let $f: \mathbb{T} \rightarrow \mathbb{R}$ be a $\Delta_{(\lambda, m)}$-measurable function and

$$
\Omega(t, m, \lambda)=\left\{s \in\left[t+m-\lambda_{t}+t_{0}-1, t+m\right)_{\mathbb{T}}: s \in \Omega\right\}
$$

for $\varepsilon>0$. In this case, we have

$$
\begin{aligned}
& \mu_{\Delta_{m}}(\Omega(t, m, \lambda)) \\
& \quad \leq \frac{1}{\varepsilon} \int_{\Omega(t, m, \lambda)}|f(s)-L| \Delta s \\
& \quad \leq \frac{1}{\varepsilon} \int_{\left[t+m-\lambda_{t}+t_{0}-1, t+m\right)_{\top}}|f(s)-L| \Delta s .
\end{aligned}
$$

Proof. This can be proved by using a method similar to the approach in [18].

Theorem 20. Let $f: \mathbb{T} \rightarrow \mathbb{R}$ be a $\Delta_{(\lambda, m)}$-measurable function, $L \in \mathbb{R}$, and $0<p<\infty$. Then, one gets the following.

(i) $\left[\widehat{W}_{m p}\right]_{\mathbb{T}} \subset \widehat{\mathbf{s}}_{\mathbb{U}}^{(\lambda, m)}$

(ii) If $f$ is $(\lambda, m)$ uniformly strongly p-summable to $L$, then $\widehat{\mathbf{s}}_{\mathbb{T}}^{(\lambda, m)}-\lim _{t \rightarrow \infty}(f(t))=L$.

(iii) If $\widehat{\mathbf{s}}_{\mathbb{\pi}}^{(\lambda, m)}-\lim _{t \rightarrow \infty}(f(t))=L$ and $f$ is a bounded function, then $f$ is uniformly strongly p-summable to L. 
Proof. (i) Let $\varepsilon>0$ and $\left[\widehat{W}_{m p}\right]_{\mathbb{T}}-\lim f(s)=L$. We can write

$$
\begin{aligned}
& \int_{\left[t+m-\lambda_{t}+t_{0}-1, t+m\right)_{\top}}|f(s)-L|^{p} \Delta s \\
& \quad \geq \int_{\Omega(t, m, \lambda)}|f(s)-L|^{p} \Delta s \geq \varepsilon^{p} \mu_{\Delta_{m}}(\Omega(t, m, \lambda)) .
\end{aligned}
$$

Therefore, $\left[\widehat{W}_{m p}\right]_{\mathbb{T}}-\lim f(s)=L$ implies $\widehat{\mathbf{s}}_{\mathbb{T}}^{(\lambda, m)}-\lim f(s)=L$.

(ii) Let $f$ be $(\lambda, m)$ uniformly strongly $p$-summable to $L$. For given $\varepsilon>0$, let

$$
\Omega(t, m, \lambda)=\left\{s \in\left[t+m-\lambda_{t}+t_{0}-1, t+m\right)_{\mathbb{T}}: s \in \Omega\right\}
$$

on time scale $\mathbb{T}$. Then, it follows from Lemma 19 that

$$
\varepsilon^{p} \mu_{\Delta_{m}}(\Omega(t, m, \lambda)) \leq \int_{\left[t+m-\lambda_{t}+t_{0}-1, t+m\right)_{\top}}|f(s)-L|^{p} \Delta s .
$$

Dividing both sides of the last inequality by $\mu_{\Delta_{(\lambda, m)}}$ ([t+m$\left.\lambda_{t}+t_{0}-1, t+m\right)_{\mathbb{T}}$ ) and taking limit as $t \rightarrow \infty$, we obtain

$$
\begin{aligned}
\lim _{t \rightarrow \infty} & \frac{\mu_{\Delta_{m}}(\Omega(t, m, \lambda))}{\mu_{\Delta_{(\lambda, m)}}\left(\left[t+m-\lambda_{t}+t_{0}-1, t+m\right)_{\mathbb{T}}\right)} \\
\leq & \frac{1}{\mathcal{E}^{p}} \lim _{t \rightarrow \infty} \frac{1}{\mu_{\Delta_{(\lambda, m)}}\left(\left[t+m-\lambda_{t}+t_{0}-1, t+m\right)_{\mathbb{T}}\right)} \\
& \times \int_{\left[t+m-\lambda_{t}+t_{0}-1, t+m\right)_{\mathbb{T}}}|f(s)-L|^{p} \Delta s=0,
\end{aligned}
$$

which yields $\widehat{\mathbf{s}}_{\mathbb{T}}^{(\lambda, m)}-\lim _{t \rightarrow \infty}(f(t))=L$.

(iii) Let $f$ be bounded and statistically convergent to $L$ on $\mathbb{T}$. Then, there exists a positive number $M$ such that $|f(s)| \leq$ $M$ for all $s \in \mathbb{T}$, and also

$$
\lim _{t \rightarrow \infty} \frac{\mu_{\Delta_{m}}(\Omega(t, m, \lambda))}{\mu_{\Delta_{(\lambda, m)}}\left(\left[t+m-\lambda_{t}+t_{0}-1, t+m\right)_{\Psi}\right)}=0,
$$

where $\Omega(t, m, \lambda)$ is as before. Since

$$
\begin{aligned}
& \int_{\left[t+m-\lambda_{t}+t_{0}-1, t+m\right)_{\top}}|f(s)-L|^{p} \Delta s \\
& =\int_{\Omega(t, m, \lambda)}|f(s)-L|^{p} \Delta s \\
& \quad+\int_{\left[t+m-\lambda_{t}+t_{0}-1, t+m\right)_{\top} / \Omega(t, m, \lambda)}|f(s)-L|^{p} \Delta s \\
& \leq(M+|L|)^{p} \int_{\Omega(t, m, \lambda)} \Delta s+\varepsilon^{p} \int_{\left[t+m-\lambda_{t}+t_{0}-1, t+m\right)_{\top}} \Delta s \\
& =(M+|L|)^{p} \mu_{\Delta_{m}}(\Omega(t, m, \lambda)) \\
& \quad+\varepsilon^{p} \mu_{\Delta_{(\lambda, m)}}\left(\left[t+m-\lambda_{t}+t_{0}-1, t+m\right)_{\Psi}\right),
\end{aligned}
$$

we obtain

$$
\begin{aligned}
\lim _{t \rightarrow \infty} & \frac{1}{\mu_{\Delta_{(\lambda, m)}}\left(\left[t+m-\lambda_{t}+t_{0}-1, t+m\right)_{\mathbb{T}}\right)} \\
& \times \int_{\left[t+m-\lambda_{t}+t_{0}-1, t+m\right)_{\mathbb{T}}}|f(s)-L|^{p} \Delta s \\
\leq & (M+|L|)^{p} \\
& \times \lim _{t \rightarrow \infty} \frac{\mu_{\Delta_{m}}(\Omega(t, m, \lambda))}{\mu_{\Delta_{(\lambda, m)}}\left(\left[t+m-\lambda_{t}+t_{0}-1, t+m\right)_{\mathbb{T}}\right)}+\varepsilon^{p} .
\end{aligned}
$$

Since $\varepsilon$ is arbitrary, the proof follows from (39) and (41).

Theorem 21. Let $f$ be a $\Delta_{m}$ measurable function. Then, $\widehat{\mathbf{s}}_{\mathbb{T}}^{(\lambda, m)}$ $\lim f(s)=L$ if and only if there exists a $\Delta_{m}$ measurable set $\Omega \subset \mathbb{T}$ such that $\delta_{\mathbb{T}}^{m}(\Omega)=1$ and $\lim _{t} f(t)=L, t \in \Omega(t, m, \lambda)$.

Proof. It can be easily proved by using similar way in the study of Turan and Duman (see [18, Theorem 3.9]).

\section{Conclusions}

In this study, we introduced the historical development of the notion of statistical convergence. Then we presented some fundamental notions based on statistical convergence. The concepts of $m$ - and $(\lambda, m)$-uniform density and uniform statistical convergence were defined on an arbitrary time scale. However, we defined $m$-uniform Cauchy functions on a time scale in general. Furthermore, we obtained some relations between these new notions.

\section{Conflict of Interests}

The authors declare that there is no conflict of interests regarding the publication of this paper.

\section{References}

[1] A. Zygmund, Trigonometric Series, Cambridge University Press, Cambridge, UK, 1979.

[2] H. Fast, "Sur la convergence statistique," Colloquium Mathematicum, vol. 2, pp. 241-244, 1951.

[3] H. Steinhaus, "Sur la convergence ordinaire et la convergence asymptotique," Colloquium Mathematicum, vol. 2, pp. 73-74, 1951.

[4] I. J. Schoenberg, "The integrability of certain functions and related summability methods. ," The American Mathematical Monthly, vol. 66, pp. 361-375, 1959.

[5] J. S. Connor, "The statistical and strong $p$-Cesàro convergence of sequences," Analysis, vol. 8, no. 1-2, pp. 47-63, 1988.

[6] J. A. Fridy, "On statistical convergence," Analysis, vol. 5, no. 4, pp. 301-313, 1985.

[7] I. J. Maddox, "Statistical convergence in a locally convex space," Mathematical Proceedings of the Cambridge Philosophical Society, vol. 104, no. 1, pp. 141-145, 1988.

[8] D. Rath and B. C. Tripathy, "On statistically convergent and statistically Cauchy sequences," Indian Journal of Pure and Applied Mathematics, vol. 25, no. 4, pp. 381-386, 1994. 
[9] T. Šalát, "On statistically convergent sequences of real numbers," Mathematica Slovaca, vol. 30, no. 2, pp. 139-150, 1980.

[10] B. C. Tripathy, "On statistical convergence," Proceedings of the Estonian Academy of Sciences Physics, vol. 47, no. 4, pp. 299-303, 1998.

[11] F. Móricz, "Statistical limits of measurable functions," Analysis, vol. 24, no. 1, pp. 1-18, 2004.

[12] R. C. Buck, “Generalized asymptotic density," American Journal of Mathematics, vol. 75, pp. 335-346, 1953.

[13] C. Belen and S. A. Mohiuddine, "Generalized weighted statistical convergence and application," Applied Mathematics and Computation, vol. 219, no. 18, pp. 9821-9826, 2013.

[14] L. Leindler, "Über die verallgemeinerte de la Vallée-Poussinsche Summierbarkeit allgemeiner Orthogonalreihen," Acta Mathematica Academiae Scientiarum Hungaricae, vol. 16, pp. 375-387, 1965.

[15] Mursaleen, “ $\lambda$-statistical convergence," Mathematica Slovaca, vol. 50, no. 1, pp. 111-115, 2000.

[16] E. Savaş, "Strong almost convergence and almost $\lambda$-statistical convergence," Hokkaido Mathematical Journal, vol. 29, no. 3, pp. 531-536, 2000.

[17] M. Seyyit Seyyidoglu and N. Ö. Tan, "A note on statistical convergence on time scale," Journal of Inequalities and Applications, vol. 2012, article 219, 2012.

[18] C. Turan and O. Duman, "Statistical convergence on timescales and its characterizations," in Advances in Applied Mathematics and Approximation Theory, vol. 41 of Springer Proceedings in Mathematics and Statistics, pp. 57-71, Springer, New York, NY, USA, 2013.

[19] S. Hilger, "Analysis on measure chains-a unified approach to continuous and discrete calculus," Results in Mathematics, vol. 18, no. 1-2, pp. 18-56, 1990.

[20] G. Sh. Guseinov, "Integration on time scales," Journal of Mathematical Analysis and Applications, vol. 285, no. 1, pp. 107-127, 2003.

[21] M. Bohner and A. Peterson, Dynamic Equations on Time Scales, an Introduction with Applications, Birkhäuser, Boston, Mass, USA, 2001.

[22] M. Bohner and G. Sh. Guseinov, "Multiple integration on time scales," Dynamic Systems and Applications, vol. 14, no. 3-4, pp. 579-606, 2005.

[23] M. Bohner and G. Sh. Guseinov, "Multiple Lebesgue integration on time scales," Advances in Difference Equations, vol. 2006, Article ID 026391, 2006.

[24] R. Agarwal, M. Bohner, D. O’Regan, and A. Peterson, "Dynamic equations on time scales: a survey," Journal of Computational and Applied Mathematics, vol. 141, no. 1-2, pp. 1-26, 2002.

[25] R. A. Raimi, "Convergence, density, and $\tau$-density of bounded sequences," Proceedings of the American Mathematical Society, vol. 14, pp. 708-712, 1963.

[26] V. Baláž and T. Šalát, "Uniform density $u$ and corresponding $I_{u^{-}}$ convergence," Mathematical Communications, vol. 11, no. 1, pp. $1-7,2006$.

[27] T. C. Brown and A. R. Freedman, "The uniform density of sets of integers and Fermat's last theorem," La Société Royale du Canada. L'Académie des Sciences, vol. 12, no. 1, pp. 1-6, 1990.

[28] I. J. Maddox, "A new type of convergence," Mathematical Proceedings of the Cambridge Philosophical Society, vol. 83, no. 1, pp. 61-64, 1978.
[29] F. Nuray, "Uniform statistical convergence," Science and Engineering Journal of Firat University, vol. 11, no. 3, pp. 219-222, 1999.

[30] D. Borwein, "Linear functionals connected with strong Cesàro summability," Journal of the London Mathematical Society, vol. 40, pp. 628-634, 1965.

[31] F. Nuray, " $\lambda$-strongly summable and $\lambda$-statistically convergent functions," Iranian Journal of Science and Technology, Transaction A: Science, vol. 34, no. 4, pp. 335-338, 2010.

[32] I. J. Maddox, "Spaces of strongly summable sequences," The Quarterly Journal of Mathematics, vol. 18, no. 2, pp. 345-355, 1967.

[33] F. Nuray and B. Aydin, "Strongly summable and statistically convergent functions," Informacines Technologijos Ir Valdymas, vol. 1, no. 30, pp. 74-76, 2004.

[34] M. Et, S. A. Mohiuddine, and A. Alotaibi, "On $\lambda$-statistical convergence and strongly $\lambda$-summable functions of order $\alpha$," Journal of Inequalities and Applications, vol. 2013, article 469, 2013.

[35] A. Cabada and D. R. Vivero, "Expression of the Lebesgue $\Delta$ integral on time scales as a usual Lebesgue integral: application to the calculus of $\Delta$-antiderivatives," Mathematical and Computer Modelling, vol. 43, no. 1-2, pp. 194-207, 2006. 


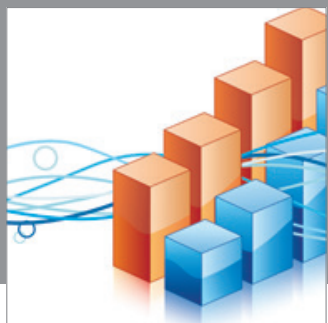

Advances in

Operations Research

mansans

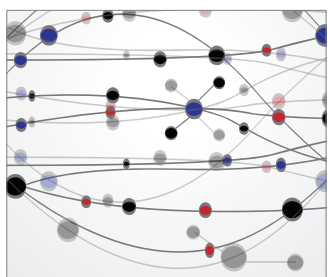

The Scientific World Journal
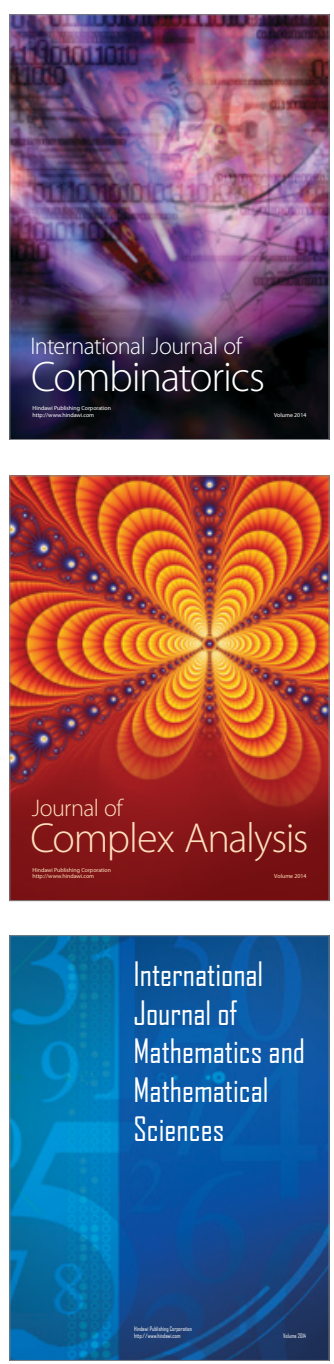
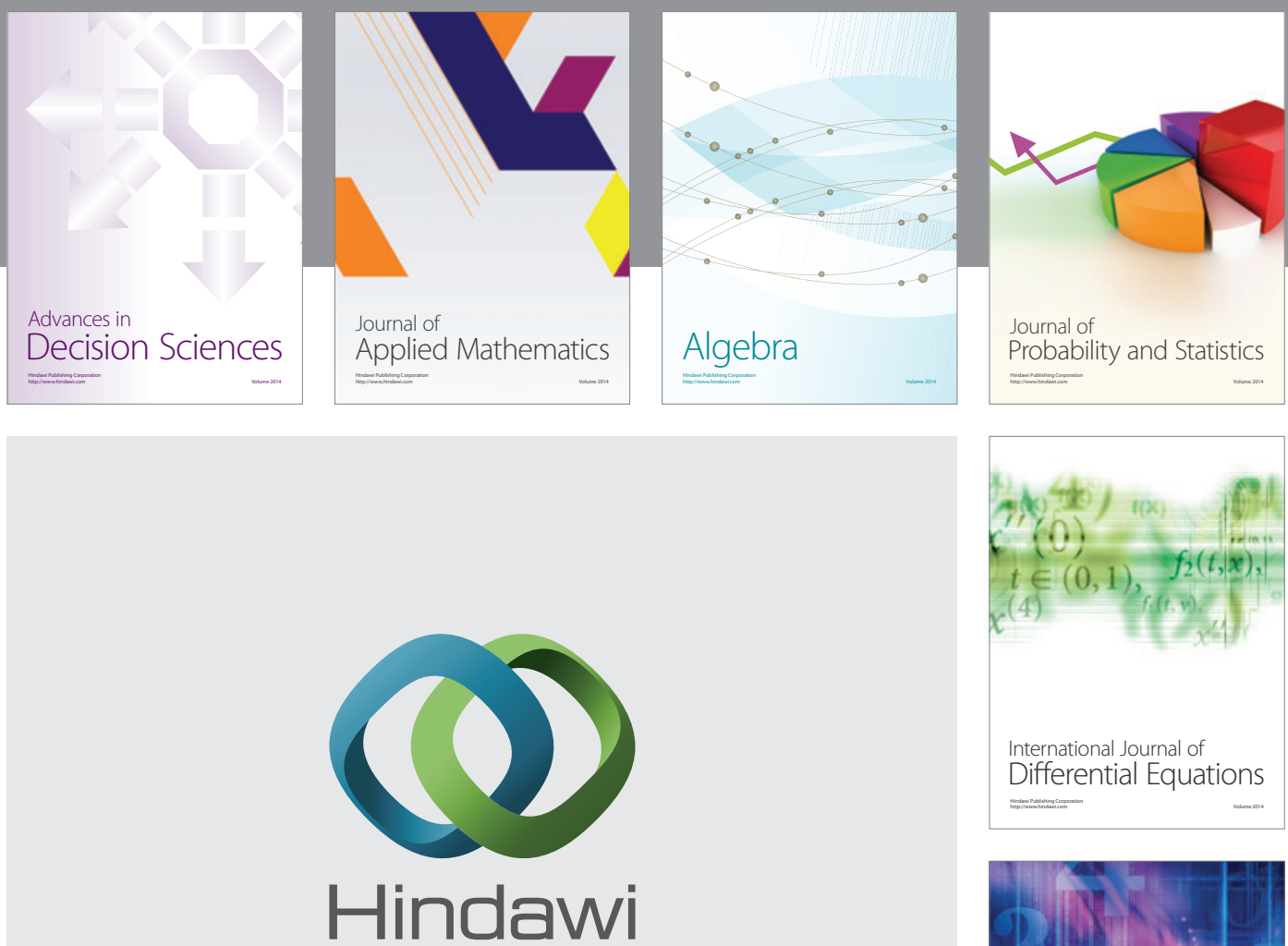

Submit your manuscripts at http://www.hindawi.com
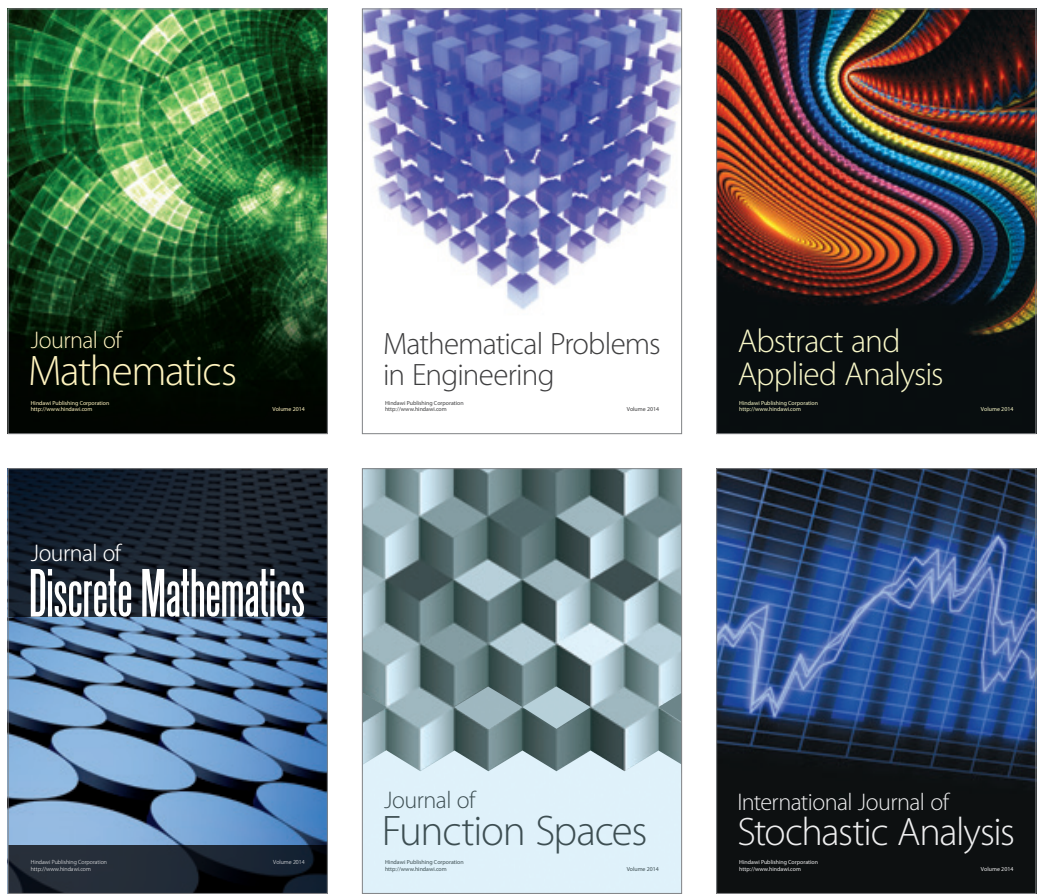

Journal of

Function Spaces

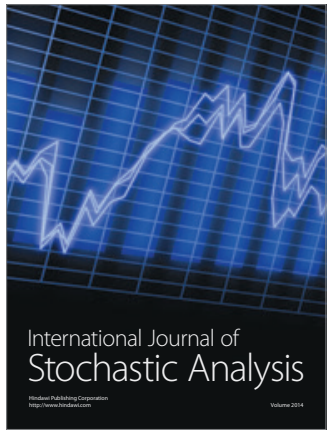

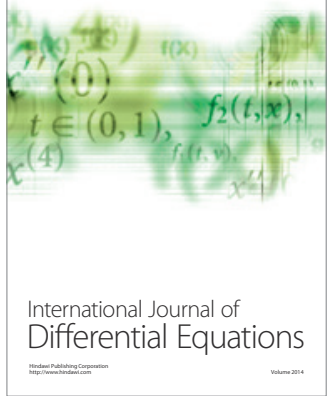
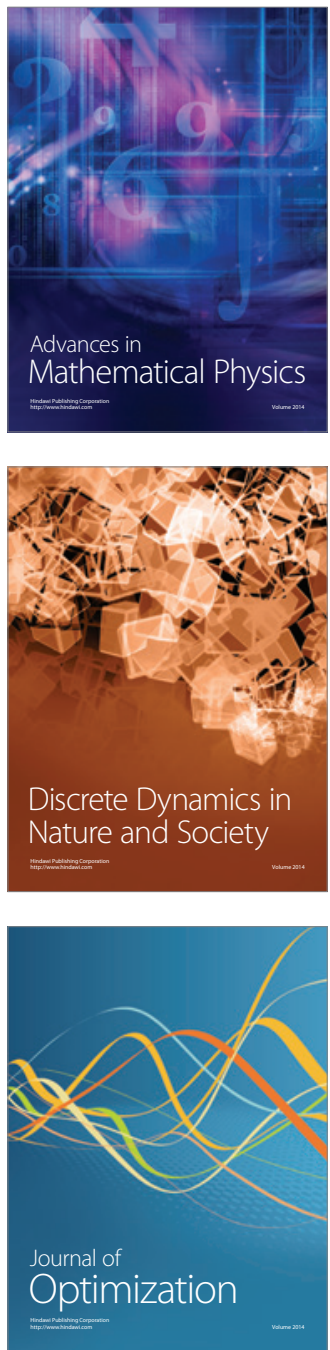